\title{
Nos normes de pratique de la pharmacie favorisent-elles les soins intégrés?
}

\author{
par Christine M Bond et Yvonne Hopf
}

¿es soins de santé devraient être sécuritaires et cliniquement travers le monde, des réformes de santé sont entreprises dans le but de faire face à l'augmentation des coûts des soins provoquée par les changements démographiques et les avancées médicales. Deux approches sont souvent utilisées pour surmonter ces défis : utiliser les compétences de l'ensemble des effectifs en soins de santé et transférer l'excédent des soins du milieu des soins secondaires au milieu des soins primaires. Au même moment, on accepte que les patients participent au processus décisionnel entourant leur santé, ce qui leur donne plus de pouvoir et se traduit par de meilleurs résultats pour leur santé. Ces réformes se sont heurtées à une opposition suscitée par des préoccupations de sécurité et la continuité des soins peut en avoir souffert. Les patients se plaignent d'avoir à répéter constamment les mêmes renseignements à propos de leur situation médicale (et sociale) à différents professionnels dont le nombre croît sans cesse.

Un concept relativement nouveau permettant d'aborder ces préoccupations est celui des soins intégrés, définis par l'Organisation mondiale de la santé comme « un concept qui rassemble les contributions, la prestation, la gestion et l'organisation de services en lien avec l'établissement de diagnostics, les traitements, les soins, la réhabilitation et la promotion de la santé. L'intégration est un moyen permettant d'améliorer les services en ce qui a trait à l'accessibilité, à la qualité, à la satisfaction des usagers et à l'efficience' ». [Traduction libre] L'intégration des soins n'est pas une entité unique, mais une approche multidimensionnelle des soins qui comprend l'intégration verticale d'un milieu à l'autre et l'intégration horizontale au sein des équipes.

Les gens qui ont les besoins les plus complexes en matière de soins de santé sont ceux qui profiteront vraisemblablement le plus des soins intégrés. Des données provenant du RoyaumeUni montrent que quatre personnes sur cinq âgées de 75 ans ou plus prennent des médicaments sur ordonnance, et $36 \%$ en prennent quatre ou plus². Les événements indésirables liés aux médicaments sont de plus en plus courants à cause des interactions médicamenteuses provoquées par la polypharmacie, des contreindications non relevées de médicaments qui ne devraient pas être prescrits à cause des comorbidités et des changements physiologiques associés au vieillissement. Tous les professionnels de la santé doivent être informés du fragile équilibre entre les risques et les avantages de l'utilisation de médicaments chez les patients qui ont des besoins complexes et les pharmaciens, en qualité d'experts dans le domaine, sont les mieux placés pour les conseiller sur la gestion sécuritaire et efficace des médicaments.

Les pharmaciens savent à quel point leur profession est indispensable pour faire en sorte que l'utilisation des médicaments soit sécuritaire et cliniquement efficace, et qu'elle ait un bon rapport coût-efficacité. Par contre, malgré le droit inaliénable du domaine de la pharmacie d'être représenté dans l'équipe centrale de santé pour fournir des soins intégrés, la plupart des projets de soins intégrés sont axés sur le rôle et la pratique des médecins; la participation d'autres professionnels de la santé au sein de l'équipe se limite souvent aux soins infirmiers.

À l'intention de nos collègues qui ne sont pas pharmaciens, voici trois exemples à l'appui du bien-fondé de nos arguments. Une importante étude récemment effectuée en Écosse montre que les pharmaciens cliniciens ont relevé et corrigé des erreurs de prescription aux fiches des médicaments de $36 \%$ des patients hospitalisés ${ }^{3}$. En moyenne, 7,5\% des ordonnances rédigées par les médecins contenaient une erreur ${ }^{3}$. Faire intervenir les pharmaciens plus tôt dans le processus de prescription et leur permettre de procéder davantage en amont à leur travail traditionnel de validation des ordonnances permettraient de prévenir de telles erreurs. Cela permettrait aussi d'éviter que ces erreurs ne soient pas détectées parce que l'effectif de la pharmacie est insuffisant pour assurer les services d'un pharmacien à toutes les unités de soins. Malgré ce constat, l'une des façons les plus utilisées pour réduire les coûts des soins de santé est de laisser des postes vacants. Les équipes de pharmacie 
sont ainsi réduites alors même que les besoins en pharmaciens sont les plus criants.

Le deuxième exemple est lié au premier. La plupart des erreurs se produisent pendant l'admission ou le départ. Bien que la méthode du bilan comparatif des médicaments soit largement reconnue comme l'une des façons de réduire le nombre d'erreurs dans la pratique, bien des défis demeurent. Les patients, particulièrement ceux dont l'hospitalisation est imprévue, peuvent parfois avoir de la difficulté à décrire quels médicaments ils prennent. De plus, un accès aux " dossiers de soins d'urgence » (un jeu de données électronique protégé par mot de passe contenant les renseignements médicaux de base de tous les patients d'Écosse et accessible seulement aux médecins et pharmaciens autorisés qui offrent des soins imprévus) n'est pas toujours possible et les pharmaciens ne sont pas toujours présents pendant l'admission.

Des études pilotes menées en Écosse ont montré les avantages du partage par voies électroniques, entre pharmaciens d'hôpitaux et pharmaciens communautaires, de renseignements portant sur les médicaments des patients au moment de l'admission et de la sortie de ceux-ci. Les patients ont donné leur accord pour l'utilisation des données à leur sujet et les pharmaciens croient que des incidents liés aux médicaments ont été évités. Il s'agit d'un excellent exemple d'intégration des soins entre les milieux qui illustre les soins axés sur le patient.

Le troisième exemple aborde la restructuration des services et l'élargissement des rôles. Le Canada et le Royaume-Uni permettent maintenant aux pharmaciens de prescrire des médicaments, une pratique particulièrement bien implantée en Écosse. Dans certains hôpitaux, la majorité des pharmaciens rédigent des ordonnances, et ce, souvent dans des domaines cliniques où une connaissance pointue des médicaments est nécessaire (par exemple en oncologie ou pour la fibrose kystique). Des preuves empiriques et des données de recherches suggèrent que l'idée du pharmacien prescripteur est accueillie favorablement et que c'est une pratique efficace. Dans une étude portant sur des pharmaciens exerçant en cabinets de médecine générale qui rédigeaient des ordonnances pour des patients souffrant de douleurs chroniques, les résultats étaient nettement meilleurs dans le groupe avec pharmaciens prescripteurs que ceux du groupe de soins habituels ${ }^{4}$. Mais, en dépit de ces résultats positifs, des contraintes financières font obstacle à la mise en œuvre plus large de cette pratique. On préfere plutôt déléguer le droit de prescrire à du personnel infirmier spécialisé, doté d'une formation moins exhaustive dans le domaine de la thérapeutique.

La majeure partie des actes des pharmaciens obéissent à des normes professionnelles. Enfin, pour conclure cet article nous examinerons de quelle manière ces normes favorisent les soins intégrés.
Les normes professionnelles canadiennes sur la pratique de la pharmacie d'hôpital' contiennent des énoncés pertinents qui sont en faveur des soins intégrés, notamment : 2.4 - être responsable de la prestation assidue de soins pharmaceutiques et de soins continus aux patients, 2.8 - collaborer avec les patients pour déterminer les besoins, pour se fixer des objectifs mutuels, et pour élaborer et exécuter un plan de soins, 2.9-coopérer avec les autres fournisseurs de soins de santé, et 4.1 - développer et entretenir un partenariat de collaboration avec les patients, les groupes de patients et les autres professionnels de la santé.

Les normes du Royaume-Uni affichent des objectifs semblables 6 . L'une des dix dimensions de soins qui s'y trouvent est « le transfert intégré des soins ». Maintenant, compte tenu du fait que les pharmaciens possèdent déjà un cadre normatif approprié, quelles actions doivent être posées pour s'assurer qu'il est traduit en un modèle de pratique permettant aux pharmaciens de jouer leur rôle dans la prestation de soins intégrés? Voici quatre suggestions d'approche.

Premièrement, il est important de générer un plus grand corpus de données scientifiques et de le disséminer. Une seule étude ne changera pas la pratique. Des études comme celles résumées ci-dessus devraient inciter d'autres travaux portant sur des contextes cliniques et géographiques différents. Deuxièmement, une formation conjointe de qualité et plus ample au premier cycle et aux cycles supérieurs est nécessaire afin d'accroître le respect mutuel entre les différentes professions et la compréhension qu'elles ont du travail de chacune. Troisièmement, les pharmaciens devraient avoir accès aux dossiers médicaux complets des patients là où cet accès n'existe pas, car, avec ces renseignements, ils pourront faire des choix éclairés par les antécédents médicaux et les traitements antérieurs, et ainsi fournir les meilleurs soins pharmaceutiques. Enfin, tous les pharmaciens doivent faire pression sur les décideurs dans le but de s'assurer que les compétences de la profession soient reconnues à leur juste valeur et afin qu'elles soient intégrées dans la planification des soins de santé. Finalement, les pharmaciens d'hôpitaux devraient se réjouir des indéniables progrès de la dernière décennie, mais il reste beaucoup à faire.

\section{Références}

1. Gröne O, Garcia-Barbero M. Trends in integrated care-reflections on conceptual issues. Copenhagen (Danemark) : Organisation mondiale de la santé; 2002. Publication $\mathrm{n}^{\circ} \mathrm{EUR} / 02 / 5037864$.

2. Quality and outwork framework: NHS employers 2012. Dans : Scottish Government prescription for excellence: a vision and action plan. Edinburgh (Royaume-Uni) : Gouvernement d'Écosse; 2013.

3. Ryan C, Ross S, Davey PG, Duncan EM, Fielding S, Francis J, et coll. Prevalence and causes of prescribing errors: the PRescribing Outcomes for Trainee doctors Engaged in Clinical Training (PROTECT) study. PLOS ONE. Sous presse.

4. Bruhn H, Bond CM, Elliott AM, Hannaford PC, Lee AJ, McNamee P, et coll. Pharmacist-led management of chronic pain in primary care: results from a randomised controlled exploratory trial. BMJ Open. 2013;3(4):e002361.

5. Normes professionnelles sur la pratique de la pharmacie d'hôpital. Ottawa $(\mathrm{ON})$ : Société canadienne des pharmaciens d'hôpitaux; 2003. 
6. Professional standards for hospital pharmacy services: optimising patient outcomes from medicines. Londres (Royaume-Uni) : Royal Pharmaceutical Society; juillet 2012. Publié au www.rpharms.com/support-pdfs/rps---professionalstandards-for-hospital-pharmacy.pdf. Consulté le 22 novembre 2013.

Christine M Bond, B. Pharm., Ph. D., M. Ed., travaille au Pharmacy, Centre of Academic Primary Care de I'Université d'Aberdeen, Foresterhill, Aberdeen, Écosse. Elle est également rédactrice adjointe du JCPH.

Yvonne Hopf, M. Pharm., M. Sc., Ph. D., travaille au Centre of Academic Primary Care de I'Université d'Aberdeen, Foresterhill, Aberdeen, Écosse. Elle est aussi pharmacienne clinicienne à la Royal Aberdeen Infirmary.

Intérêts concurrents : Christine Bond a reçu le remboursement de ses frais de déplacement par Wiley pour sa présence à des conférences et à des réunions professionnelles en tant que rédactrice en chef de I'International Journal of Pharmacy Practice, ainsi que par les Instituts de recherche en santé du Canada pour sa participation aux « Échanges Meilleurs cerveaux de 2012 ». Elle a obtenu une multitude de subventions, versées à son établissement, de la part de conseils de recherche et d'associations caritatives d'Europe, du Royaume-Uni et d'Écosse de même que de Novartis pour des activités de recherche effectuées hors du cadre de cet article. Yvonne Hopf a reçu une National Health Service Applied Programme Grant et une bourse de recherche postdoctorale du Chief Scientist Office d'Écosse pour des travaux effectués hors du cadre de cet article.

\section{Adresse de correspondance :}

$D^{\text {re }}$ Christine M Bond

Pharmacy, Centre of Academic Primary Care Room 1.123, Polwarth Building West Block Foresterhill, Aberdeen AB25 2ZD

Scotland

Courriel : c.m.bond@abdn.ac.uk 